TRANSACTIONS OF THE

AMERICAN MATHEMATICAL SOCIETY

Volume 351 , Number 12, Pages 4847-4860

S 0002-9947(99)02508-8

Article electronically published on August 30, 1999

\title{
STEEPEST DESCENT EVOLUTION EQUATIONS: ASYMPTOTIC BEHAVIOR OF SOLUTIONS AND RATE OF CONVERGENCE
}

\author{
R. COMINETTI AND O. ALEMANY
}

\begin{abstract}
We study the asymptotic behavior of the solutions of evolution equations of the form $\dot{u}(t) \in-\partial f(u(t), r(t))$, where $f(\cdot, r)$ is a one-parameter family of approximations of a convex function $f(\cdot)$ we wish to minimize.

We investigate sufficient conditions on the parametrization $r(t)$ ensuring that the integral curves $u(t)$ converge when $t \rightarrow \infty$ towards a particular minimizer $u_{\infty}$ of $f$. The speed of convergence is also investigated, and a result concerning the continuity of the limit point $u_{\infty}$ with respect to the parametrization $r(\cdot)$ is established.

The results are illustrated on different approximation methods. In particular, we present a detailed application to the logarithmic barrier in linear programming.
\end{abstract}

\section{INTRODUCTION}

Let $f: H \rightarrow \mathbb{R} \cup\{+\infty\}$ be a closed proper convex function defined on a real Hilbert space $H$, and consider the minimization problem

$$
\min \{f(x): x \in H\} \text {. }
$$

It is known (see [4, Brézis], [5, Bruck]) that when the minimum in $(P)$ is attained, the trajectory defined by the steepest descent method

$$
\left\{\begin{array}{l}
\dot{u}(t) \in-\partial f(u(t)) \\
u(0)=u_{0}
\end{array}\right.
$$

converges weakly as $t \rightarrow \infty$, towards an optimal solution $u_{\infty}$ of $(P)$. Despite the theoretical importance of this result, the numerical approximation of the trajectory $u(t)$ may be difficult because of the multivalued character of the operator $\partial f$ and the fact that $\partial f(x)$ may be empty. Moreover, when $(P)$ has multiple solutions, the result does not provide additional information on which specific optimum $u_{\infty}$ will be attained in the limit.

To overcome these drawbacks, one may combine the steepest descent idea with regularization techniques by considering a one-parameter family of approximating

Received by the editors February 5, 1997.

1991 Mathematics Subject Classification. Primary 34C35, 34D05; Secondary 49M10, 49M30.

Key words and phrases. Dissipative evolution equations, steepest descent, penalty and viscosity methods, convex optimization.

This work was completed while the first author was visiting Laboratoire d'Econometrie, Ecole Polytechnique, Paris. Partially supported by Comisión Nacional de Investigación Científica y Tecnológica de Chile under Fondecyt grant 1961131.

(C)1999 American Mathematical Society 
problems of the form

$$
\min \{f(x, r): x \in H\},
$$

where the functions $f(\cdot, r)$ are more regular than $f$ in terms of smoothness and/or strict convexity, and converge to the latter in an appropriate variational sense as $r \rightarrow 0$. In this setting one is led to combine the approximation scheme with the steepest descent method by considering the non-autonomous evolution equation

$$
\left\{\begin{array}{l}
\dot{u}(t) \in-\partial f(u(t), r(t)) \\
u(0)=u_{0}
\end{array}\right.
$$

where $r:[0,+\infty) \rightarrow(0,+\infty)$ is a decreasing function such that $\lim _{t \rightarrow \infty} r(t)=0$.

This approach has already been considered in [2], where convergence results for the integral curves of $(E V)$ have been established under a strong convexity assumption on the approximating functions $f(\cdot, r)$, and assuming that the parametrization $r(t)$ decays slowly towards 0. Our first goal in this paper is to extend these results by studying the rate of convergence of the integral curves of $(E V)$. These results are used in the context of approximation schemes for linear programming, in order to establish the convergence of some dual paths associated with the integral curves $u(t)$, towards dual optimal solutions.

In the case of a parametrization $r(t)$ which decays fast towards 0 , the asymptotic convergence of $u(t)$ has been investigated in [9] for the general evolution equation $(E V)$, and also in [7] in the particular setting of the exponential penalty method for linear programming. In $\S 4$ we generalize the results of [7] to the abstract setting of equation $(E V)$.

The results will be illustrated in $\S 5$ by considering a detailed analysis of the specific example given by the logarithmic barrier in linear programming.

The assumptions of slow and fast decay for $r(t)$ are not always complementary, and there may be a gap between them. However, when these conditions are complementary, that is to say, when the integral curves $u(t)$ converge for every parametrization $r(t)$, we shall prove that the limit point varies continuously when passing from the case of fast decay to the case of slow decay. This continuity result will be established in the final section, $\S 6$.

In the sequel we shall freely use the standard results and notations of convex analysis, for which the reader is referred to the classical references [8, 14].

\section{BASIC ASSUMPTIONS}

Throughout this paper we consider a real Hilbert space $H$ with scalar product and norm denoted by $\langle\cdot, \cdot\rangle$ and $|\cdot|$ respectively, while $f: H \rightarrow \mathbb{R} \cup\{+\infty\}$ will be a closed proper convex function such that the minimization problem

$$
\min \{f(x): x \in H\}
$$

has a nonempty set of optimal solutions $S(P) \neq \emptyset$.

We also consider a one-parameter family $\{f(\cdot, r): r>0\}$ of closed proper convex functions defined on $H$, such that the minimum

$$
\min \{f(x, r): x \in H\}
$$


is an approximation of $(P)$ in the following sense:

$(H)\left\{\begin{array}{l}\min _{x} f(x, r) \rightarrow \min _{x} f(x) \text { when } r \rightarrow 0, \text { and there exists a trajectory of } \\ \text { optimal solutions } x(r) \text { of }\left(P_{r}\right) \text { which is absolutely continuous on every } \\ \text { compact interval }\left[r_{1}, r_{2}\right] \text { with } 0<r_{1} \leq r_{2} \text {, and converges (strongly) to- } \\ \text { wards some } x^{*} \in S(P) \text { when } r \rightarrow 0 .\end{array}\right.$

We let $\gamma:(0,+\infty) \rightarrow(0,+\infty)$ be a continuous function such that

$$
\left|\frac{d x}{d r}(r)\right| \leq \frac{1}{\gamma(r)} \quad \text { a.e. } r>0 .
$$

Without loss of generality we assume that $\gamma$ is bounded, but we allow $\gamma(r) \rightarrow 0$ as $r \rightarrow 0$, so that the derivative $\frac{d x}{d r}(r)$ may eventually explode as $r \rightarrow 0$.

We associate with $\left(P_{r}\right)$ the non-autonomous evolution equation

$$
\left\{\begin{array}{l}
\dot{u}(t) \in-\partial f(u(t), r(t)) \\
u(0)=u_{0}
\end{array}\right.
$$

where $r:[0,+\infty) \rightarrow(0,+\infty)$ is a differentiable decreasing function such that

$$
\lim _{t \rightarrow \infty} r(t)=0 \text {. }
$$

We assume that $(E V)$ has a (necessarily unique) global solution $u:[0,+\infty) \rightarrow H$, that is to say, an absolutely continuous function such that $\dot{u}(t) \in-\partial f(u(t), r(t))$ a.e. in $(0,+\infty)$ and $u(0)=u_{0}$. The existence of global solutions for differential inclusions like the previous one has been extensively studied in a number of papers (see e.g. [1, Attouch \& Damlamian], [10, Kenmochi] and references therein).

\section{Slow parametrization AND RATE OF CONVERGENCE}

A general approach to the asymptotic analysis of the solution $u(t)$ of $(E V)$ was given in [2], based on the assumption $(H)$ and the slow decay of the parametrization $r(t)$ expressed by the condition

$$
\int_{0}^{\infty} \beta(r(\tau)) d \tau=+\infty
$$

where $\beta(r)$ is a strong convexity constant for the function $f(\cdot, r)$, that is to say,

$$
\left\{\begin{array}{l}
\text { for all } r>0 \text { there exists } \beta(r)>0 \text { such that } \\
f(x, r)+\langle v, y-x\rangle+\frac{\beta(r)}{2}|x-y|^{2} \leq f(y, r) \\
\text { for every } x, y \in H \text { and } v \in \partial f(x, r) .
\end{array}\right.
$$

More precisely, we have

Theorem 3.1. In addition to the basic assumptions in $\S 2$ let us suppose that $(S C)$ holds with $\beta(\cdot)$ measurable and bounded over bounded sets, and that the parametrization $r(t)$ satisfies (1) and

$$
\lim _{t \rightarrow \infty} \frac{\dot{r}(t)}{\gamma(r(t)) \beta(r(t))}=0 .
$$

Then $u(t)$ converges strongly towards $x^{*}$ as $t \rightarrow \infty$.

Proof. See [2, Theorem 3.3]. 
The essence of the analysis in such a setting consists in proving that

$$
\lim _{t \rightarrow \infty}|u(t)-x(r(t))|=0,
$$

which combined with $(H)$ shows that $u(t)$ is attracted by $x(r(t))$ towards $x^{*} \in S(P)$. Intuitively, as the parametrization $r(t)$ goes to 0 slower and slower, the attraction towards the optimal path $x(r(t))$ has enough time to act, and $|u(t)-x(r(t))|$ will tend to zero faster and faster compared to $r(t)$. We give a formal statement of this fact by studying the rate at which $u(t)$ approaches the optimal trajectory $x(r(t))$.

Theorem 3.2. In addition to the basic assumptions in $\S 2$, let us suppose that $(S C)$ holds with $\beta(\cdot)$ measurable and bounded over bounded sets. Let $k \geq 1$, and let $r(t)$ be a parametrization such that

$$
\lim _{t \rightarrow \infty} \frac{\dot{r}(t)}{\gamma(r(t)) \beta(r(t)) r(t)^{k}}=0 .
$$

Then

$$
\lim _{t \rightarrow \infty} \frac{|u(t)-x(r(t))|}{r(t)^{k}}=0 .
$$

Proof. Let $E(t):=\int_{0}^{t} \beta(r(\tau)) d \tau$. As in [2, Theorem 3.2] it can be proved that

$$
\exp [E(t)]|u(t)-x(r(t))| \leq|u(0)-x(r(0))|-\int_{0}^{t} \exp [E(\tau)]\left|\frac{d x}{d r}(r(\tau))\right| \dot{r}(\tau) d \tau,
$$

from which it follows that (notice that $\dot{r}(t) \leq 0$ )

$$
\frac{|u(t)-x(r(t))|}{r(t)^{k}} \leq \frac{|u(0)-x(r(0))|}{\exp [E(t)] r(t)^{k}}-\frac{\int_{0}^{t} \exp [E(\tau)] \dot{r}(\tau) / \gamma(r(\tau)) d \tau}{\exp [E(t)] r(t)^{k}} .
$$

We claim that $\delta_{k}(t):=\exp [E(t)] r(t)^{k}$ diverges to $+\infty$ as $t \rightarrow \infty$, so the first term on the right hand side of (4) goes to 0 . Indeed, a straightforward computation gives

$$
\frac{d}{d t}\left[\delta_{k+1}(t)\right]=\beta(r(t)) \delta_{k+1}(t)\left[1+(k+1) \frac{\dot{r}(t)}{\beta(r(t)) r(t)}\right] .
$$

The boundedness of $\gamma(\cdot)$ together with (3) implies that

$$
\lim _{t \rightarrow \infty} \frac{\dot{r}(t)}{\beta(r(t)) r(t)}=0
$$

so that $\frac{d}{d t} \delta_{k+1}(t)>0$ for $t$ sufficiently large. Hence $\delta_{k+1}(t)$ is increasing for $t$ large, and we may select $t_{0}$ such that $\delta_{k+1}(t) \geq \delta_{k+1}\left(t_{0}\right)$ for $t \geq t_{0}$. This implies that $\exp [E(t)] r(t)^{k} \geq \exp \left[E\left(t_{0}\right)\right] r\left(t_{0}\right)^{k+1} / r(t) \rightarrow+\infty$, as claimed.

It remains to show that the second term on the right hand side of (4) also tends to 0 . We shall do this by using l'Hôpital's rule.

Let us begin by checking that $\Gamma(t):=-\int_{0}^{t}(\exp [E(\tau)] \dot{r}(\tau) / \gamma(r(\tau))) d \tau$ diverges to $+\infty$ as $t \rightarrow \infty$. To this end let $g(t):=-\int_{0}^{t} \exp [E(\tau)] \dot{r}(\tau) d \tau$ and $\psi(t):=$ $\exp [E(t)] r(t)$. Using (5) we get

$$
\frac{\dot{g}(t)}{\dot{\psi}(t)}=\frac{-\exp [E(t)] \dot{r}(t)}{\exp [E(t)](\beta(r(t)) r(t)+\dot{r}(t))}=\frac{-\dot{r}(t) /[\beta(r(t)) r(t)]}{1+\dot{r}(t) /[\beta(r(t)) r(t)]} \longrightarrow 0
$$


as $t \rightarrow \infty$. Therefore we may find $T>0$ with $\dot{g}(t) \leq \dot{\psi}(t)$ for all $t \geq T$, so that $(g-\psi)$ is decreasing, and then

$$
g(t)-\psi(t) \leq g(T)-\psi(T) \text { for all } t \geq T .
$$

Now, letting $h(t):=r(t)(g(t)-g(T))$ and using the previous inequality, we get

$$
\begin{aligned}
\dot{h}(t) & =\dot{r}(t)(g(t)-g(T))+r(t) \dot{g}(t) \\
& =\dot{r}(t)(g(t)-g(T))-\exp [E(t)] \dot{r}(t) r(t) \\
& =\dot{r}(t)(g(t)-g(T)-\psi(t)) \\
& \geq-\dot{r}(t) \psi(T) \\
& \geq 0 .
\end{aligned}
$$

Hence $h(t)$ is increasing, so that for $t>\bar{t}>T$ we have $h(t) \geq h(\bar{t})$. Thus

$$
g(t) \geq g(T)+\frac{r(\bar{t})}{r(t)}(g(\bar{t})-g(T)) .
$$

Since $g(\bar{t})>g(T)$ for $\bar{t}>T$ and $r(t) \rightarrow 0$, it follows that $g(t) \rightarrow+\infty$ when $t \rightarrow \infty$. Comparing the expressions of $g(t)$ and $\Gamma(t)$, and using the boundedness of $\gamma(\cdot)$, we conclude that $\lim _{t \rightarrow \infty} \Gamma(t)=+\infty$ as well.

We complete the proof by showing that the second term on the right hand side of (4) tends to 0 . Indeed, a straightforward computation gives

$$
\frac{\dot{\Gamma}(t)}{\dot{\delta}_{k}(t)}=\frac{-\dot{r}(t) /\left[\gamma(r(t)) \beta\left(r(t) r(t)^{k}\right]\right.}{1+k \dot{r}(t) /[\beta(r(t)) r(t)]},
$$

which converges to 0 by (3) and (5). Using l'Hôpital's rule, we deduce that

$$
\lim _{t \rightarrow \infty} \frac{-\int_{0}^{t} \exp [E(\tau)] \dot{r}(\tau) / \gamma(r(\tau)) d \tau}{\exp [E(t)] r(t)^{k}}=\lim _{t \rightarrow \infty} \frac{\Gamma(t)}{\delta_{k}(t)}=\lim _{t \rightarrow \infty} \frac{\dot{\Gamma}(t)}{\dot{\delta}_{k}(t)}=0,
$$

as was to be proved.

Remark 3.1. Notice that, in contrast with Theorem 3.1, condition (1) is not assumed in Theorem 3.2. As a matter of fact, as a by-product of the above proof $\left(\delta_{k}(t) \rightarrow \infty\right.$ as $\left.t \rightarrow \infty\right)$, we obtain that (1) is a consequence of (3) provided $k \geq 1$. We do not know if (1) also follows from this condition when $k=0$, but one can show that this is the case if one assumes in addition that $\int_{0}^{r_{0}}(1 / \gamma(r)) d r=+\infty$.

The strict convexity condition $(S C)$ may be too stringent for some approximation schemes (we shall see this in the examples), while a localized version of this condition may still hold, namely

$$
\left\{\begin{array}{l}
\text { for all } r>0 \text { and } K>0 \text { there exists } \beta_{K}(r)>0 \text { such that } \\
f(x, r)+\langle v, y-x\rangle+\frac{\beta_{K}(r)}{2}|x-y|^{2} \leq f(y, r) \\
\text { for every } x, y \in H \text { such that }|x| \leq K,|y| \leq K, \text { and } v \in \partial f(x, r) .
\end{array}\right.
$$

Based on this relaxed condition, one can get the following variant of Theorem 3.1.

Theorem 3.3. In addition to the basic assumptions in $\S 2$ let us suppose that the optimal trajectory $x(r)$ has finite length,

$$
\int_{0}^{r_{0}}\left|\frac{d x}{d r}(r)\right| d r<+\infty \text { for some } r_{0}>0 .
$$


Let us assume also that $(L S C)$ holds with $\beta_{K}(\cdot)$ measurable and bounded over bounded sets, and that the parametrization $r(t)$ satisfies

$$
\int_{0}^{\infty} \beta_{K}(r(\tau)) d \tau=+\infty \text { for all } K>0
$$

Then $u(t)$ converges strongly towards $x^{*}$ as $t \rightarrow \infty$.

Proof. See [2, Theorem 3.2].

The finite length assumption, which replaces condition (2) of Theorem 3.1, is used to establish that the solution $u(t)$ remains bounded (see the proof of $[2$, Theorem 3.2]). Hence there exists a constant $K>0$ such that the trajectories $u(t)$ and $x(r(t))$ remain in $B(0, K)$, and one may repeat the analysis of Theorem 3.2 with $\beta_{K}(r)$ in place of the global strong convexity constant $\beta(r)$. Taking this observation into account, we may state the following variant of Theorem 3.2.

Theorem 3.4. In addition to the basic assumptions in $\S 2$, let us suppose that the finite length hypothesis $(6)$ and $(L S C)$ hold with $\beta_{K}(\cdot)$ measurable and bounded over bounded sets. Let $k \geq 1$, and let $r(t)$ be a parametrization such that

$$
\lim _{t \rightarrow \infty} \frac{\dot{r}(t)}{\gamma(r(t)) \beta_{K}(r(t)) r(t)^{k}}=0 \text { for all } K>0 \text {. }
$$

Then

$$
\lim _{t \rightarrow \infty} \frac{|u(t)-x(r(t))|}{r(t)^{k}}=0 .
$$

The previous results on the rate of convergence will be exploited in $\S 5$ in the context of the duality theory for the logarithmic barrier method in linear programming. For the moment we present two simple applications.

3.1. Example: the viscosity method. Let us consider the original problem $(P)$ with an optimal set $S(P) \neq \emptyset$ not reduced to a singleton. The classical Tikhonov regularization scheme [15] approximates $(P)$ by the family of strongly convex functions $f(x, r)=f(x)+\frac{r}{2}|x|^{2}$, for which $(S C)$ is satisfied with $\beta(r)=r$.

It is known [15] that in this case $\left(P_{r}\right)$ has a unique optimal solution $x(r)$ which converges to the element $x^{*} \in S(P)$ with minimal norm. Moreover, $\left|\frac{d x}{d r}(r)\right| \leq C / r$ for some constant $C>0$ (see $[2,16]$ ), so we can take $\gamma(r)=r$.

The application of Theorem 3.2 is immediate: if we choose a parametrization $r(t)$ such that $\lim _{t \rightarrow \infty} \dot{r}(t) / r(t)^{k+2}=0$ with $k \geq 1$ (for instance $r(t)=1 / t^{\alpha}$ with $\alpha<1 /(k+1))$, then the solution $u(t)$ of

$$
\left\{\begin{array}{l}
\dot{u}(t)+\partial f(u(t))+r(t) u(t)=0 \\
u(0)=u_{0}
\end{array}\right.
$$

satisfies

$$
\lim _{t \rightarrow \infty} \frac{|u(t)-x(r(t))|}{r(t)^{k}}=0 .
$$

Furthermore, by using Theorem 3.1 and noting that $\lim _{t \rightarrow \infty} \dot{r}(t) / r(t)^{2}=0$ implies $\int_{0}^{\infty} r(\tau) d \tau=+\infty$, it follows easily that this remains true for $k=0$. 
3.2. Example: the exponential penalty. Consider the linear program

$$
\min _{x \in \mathbb{R}^{n}}\left\{c^{t} x: A x \leq b\right\}
$$

where $c \in \mathbb{R}^{n}, A$ is an $m \times n$ matrix, and $b \in \mathbb{R}^{m}$. We assume that $(L P)$ has a nonempty and bounded optimal solution set $S(L P) \neq \emptyset$. Recall that the dual problem of $(L P)$ is given by

$$
\min _{\lambda \in \mathbb{R}^{m}}\left\{b^{t} \lambda: A^{t} \lambda+c=0, \lambda \geq 0\right\} .
$$

The exponential penalty method consists in approximating $(L P)$ by the smooth unconstrained problems

$$
\min _{x \in \mathbb{R}^{n}} c^{t} x+r \sum_{i=1}^{m} \exp \left[\left(A_{i} x-b_{i}\right) / r\right],
$$

where $A_{i}$ denote the rows of $A$, so that in this case

$$
f(x, r)=c^{t} x+r \sum_{i=1}^{m} \exp \left[\left(A_{i} x-b_{i}\right) / r\right] .
$$

It is known [7] that $\left(L P_{r}\right)$ has a unique optimal solution $x(r)$ and that this trajectory converges when $r \rightarrow 0$ towards a particular point $x^{*} \in S(L P)$ called the centroid. Moreover $x(r)$ is of class $C^{\infty}$ on $(0,+\infty)$ and $\left|\frac{d x}{d r}(r)\right|$ remains bounded as $r \rightarrow 0$, so that the trajectory has finite length.

A direct computation of the Hessian of $f(\cdot, r)$ shows that $(L S C)$ is satisfied, but $(S C)$ fails unless the feasible set of $(L P)$ is bounded. More precisely, for every $K>0$ there exist positive constants $M$ and $L$ such that $\beta_{K}(r) \geq(L / r) \exp [-M / r]$.

The existence (and uniqueness) of a global solution $u(t)$ for $(E V)$ in the case of the exponential penalty was established in [6]. In the same reference it is proved (this also follows from Theorem 3.3) that under the assumption of slow decay

$$
\int_{0}^{\infty} \frac{1}{r(\tau)} \exp (-\alpha / r(\tau)) d \tau=\infty \text { for all } \alpha>0
$$

the trajectory $u(t)$ converges towards the centroid $x^{*}$.

The application of Theorem 3.4 to this example is straightforward. Since $\left|\frac{d x}{d r}(r)\right|$ is bounded, if we choose the parametrization $r(t)$ so that

$$
\lim _{t \rightarrow \infty} \frac{\exp (\alpha / r(t)) \dot{r}(t)}{r(t)^{k-1}}=0 \text { for all } \alpha>0
$$

with $k \geq 1$ (this holds for instance with $r(t)=1 / \ln (\ln t)$ for every $k \geq 1$ ), then

$$
\lim _{t \rightarrow \infty} \frac{|u(t)-x(r(t))|}{r(t)^{k}}=0 .
$$

In the following result the case $k=1$ plays an important role.

Corollary 3.5 (Convergence of Multipliers). For all $i \in I$ let us set

$$
\lambda_{i}(t):=\exp \left[\left(A_{i} u(t)-b_{i}\right) / r(t)\right] .
$$

If $r(t)$ satisfies

$$
\lim _{t \rightarrow \infty} \exp (\alpha / r(t)) \dot{r}(t)=0 \text { for all } \alpha>0,
$$

then $\lim _{t \rightarrow \infty} \lambda_{i}(t)$ exists, and it is an optimal solution of the dual problem $(D P)$. 
Proof. It is known (see [6]) that $\left(\exp \left[\left(A_{i} x(r)-b_{i}\right) / r\right] ; i \in I\right)$ converges when $r \rightarrow 0$ towards a dual optimal solution $\lambda^{*} \in S(D P)$. Since by the previous discussion we have

$$
\lim _{t \rightarrow \infty} \frac{|u(t)-x(r(t))|}{r(t)}=0,
$$

it follows at once that $\left(\exp \left[\left(A_{i} u(t)-b_{i}\right) / r(t)\right] ; i \in I\right)$ converges to the same $\lambda^{*}$.

\section{Fast convergence Results}

In the setting of the exponential penalty of example 3.2 , it was proved in [6] that under the assumption of fast decay

$$
\int_{0}^{\infty} r(\tau) \exp (-M / r(\tau)) d \tau<\infty \text { for all } M>0,
$$

the trajectory $u(t)$ still converges towards a particular solution $u_{\infty} \in S(P)$, which may now be different from the centroid $x^{*}$. In this section we extend this result to the general setting of $(E V)$, for which we shall use the following lower semicontinuity assumption:

$$
f(x) \leq \liminf _{k \rightarrow \infty} f\left(x_{k}, r_{k}\right) \text { for all } x \in H, r_{k} \rightarrow 0 \text { and } x_{k} \rightarrow x .
$$

We distinguish the finite and infinite dimensional cases.

Theorem 4.1. In addition to the basic assumptions in $\S 2$, let us suppose that $(C)$ holds, that $H$ is finite dimensional and that the optimal trajectory $x(r)$ satisfies the finite length assumption (6). Let $r(t)$ be such that

$$
\text { for all } \bar{u} \in S(P) \text { there exists } \epsilon>0 \text { such that } \lim _{t \rightarrow \infty} \Gamma_{\epsilon}(t)<\infty \text {, }
$$

where $\Gamma_{\epsilon}(t):=\int_{0}^{t}\left[f\left(x(r(\tau))+\epsilon\left(\bar{u}-x^{*}\right), r(\tau)\right)-f(x(r(\tau)), r(\tau))\right] d \tau$. Then the solution $u(t)$ of $(E V)$ converges to a particular solution $u_{\infty} \in S(P)$.

Proof. We claim that $|u(t)-\bar{u}|$ converges when $t \rightarrow \infty$, for all $\bar{u} \in S(P)$. The conclusion will follow by showing the existence of a sequence $t_{k} \rightarrow \infty$ such that $u\left(t_{k}\right)$ converges towards a point $u_{\infty} \in S(P)$, so that

$$
\lim _{t \rightarrow \infty}\left|u(t)-u_{\infty}\right|=\lim _{k \rightarrow \infty}\left|u\left(t_{k}\right)-u_{\infty}\right|=0
$$

We begin by considering the specific case $\bar{u}=x^{*}$.

Let $\varphi(t):=\frac{1}{2}|u(t)-x(r(t))|^{2}$ and $\Delta(t):=\langle-\dot{u}(t), u(t)-x(r(t))\rangle$. A straightforward computation gives, for almost all $t>0$,

$$
\begin{aligned}
\dot{\varphi}(t) & =\left\langle\dot{u}(t)-\dot{r}(t) \frac{d x}{d r}(r(t)), u(t)-x(r(t))\right\rangle \\
& \leq-\Delta(t)-\dot{r}(t)\left|\frac{d x}{d r}(r(t))\right||u(t)-x(r(t))| .
\end{aligned}
$$

We observe that since $-\dot{u}(t) \in \partial f(u(t), r(t))$ a.e. and $0 \in \partial f(x(r(t)), r(t))$, the monotonicity of the subdifferential implies that $\Delta(t) \geq 0$ a.e., and then

$$
\dot{\varphi}(t) \leq-\dot{r}(t)\left|\frac{d x}{d r}(r(t))\right||u(t)-x(r(t))| \leq-\dot{r}(t)\left|\frac{d x}{d r}(r(t))\right| \sqrt{2(\varphi(t)+1)} .
$$


Dividing by $2 \sqrt{\varphi(t)+1}$ and defining $I(t):=\frac{1}{\sqrt{2}} \int_{r(t)}^{r(0)}\left|\frac{d x}{d r}\right| d r$, we deduce that

$$
\frac{d}{d t}[\sqrt{\varphi(t)+1}-I(t)] \leq 0 \text { a.e., }
$$

so that $\sqrt{\varphi(t)+1}-I(t)$ is non-increasing. Now, the convergence of $I(t)$ implies that $\sqrt{\varphi(t)+1}-I(t)$ is bounded below, so that it converges, and then $\varphi(t)$ converges too. Since $x(r(t))$ converges to $x^{*}$, we conclude the existence of $\lim _{t \rightarrow \infty}\left|u(t)-x^{*}\right|$.

Let us consider next the convergence of $|u(t)-\bar{u}|$ for an arbitrary $\bar{u} \in S(P)$. Since

$$
|u(t)-\bar{u}|^{2}=\left|u(t)-x^{*}\right|^{2}+\left|x^{*}-\bar{u}\right|^{2}-2\left\langle x^{*}, x^{*}-\bar{u}\right\rangle+2\left\langle u(t), x^{*}-\bar{u}\right\rangle,
$$

it suffices to establish the existence of $\lim _{t \rightarrow \infty}\left\langle u(t), x^{*}-\bar{u}\right\rangle$. In order to show this, we use Fenchel's inequality and the inclusion $-\dot{u}(t) \in \partial f(u(t), r(t))$ to get

$$
\left\langle-\dot{u}(t), x(r(t))+\epsilon\left(\bar{u}-x^{*}\right)-u(t)\right\rangle \leq f\left(x(r(t))+\epsilon\left(\bar{u}-x^{*}\right), r(t)\right)-f(u(t), r(t)),
$$

so that, using the optimality of $x(r(t))$ and the definition of $\Delta(t)$, we deduce

$$
\epsilon\left\langle\dot{u}(t), x^{*}-\bar{u}\right\rangle \leq \Delta(t)+f\left(x(r(t))+\epsilon\left(\bar{u}-x^{*}\right), r(t)\right)-f(x(r(t)), r(t)),
$$

which implies that

$$
\frac{d}{d t}\left[\epsilon\left\langle u(t), x^{*}-\bar{u}\right\rangle-\int_{0}^{t} \Delta(\tau) d \tau-\Gamma_{\epsilon}(t)\right] \leq 0 .
$$

From the hypothesis of the theorem we have that $\Gamma_{\epsilon}(t)$ converges as $t \rightarrow \infty$. On the other hand, using (9) and the boundedness of $\varphi(t)$, we may find a constant $L \geq 0$ such that

$$
0 \leq \Delta(t) \leq-\dot{\varphi}(t)-L \dot{r}(t)\left|\frac{d x}{d r}(r(t))\right|,
$$

which after integration yields

$$
\begin{aligned}
\int_{0}^{t} \Delta(\tau) d \tau & \leq \varphi(0)-\varphi(t)+L \int_{r(t)}^{r(0)}\left|\frac{d x}{d r}\right| d r \\
& \leq \varphi(0)+L \int_{0}^{r(0)}\left|\frac{d x}{d r}\right| d r
\end{aligned}
$$

from which we also obtain the convergence of $\int_{0}^{t} \Delta(\tau) d \tau$ when $t \rightarrow \infty$.

These convergences together with the boundedness of $u(t)$ imply that the function $t \rightarrow \epsilon\left\langle u(t), x^{*}-\bar{u}\right\rangle-\int_{0}^{t} \Delta(\tau) d \tau-\Gamma_{\epsilon}(t)$ is non-increasing and bounded below, thus convergent when $t \rightarrow \infty$. From this it follows at once that $\left\langle u(t), x^{*}-\bar{u}\right\rangle$ converges, establishing our claim: $|u(t)-\bar{u}|$ converges when $t \rightarrow \infty$, for all $\bar{u} \in S(P)$.

It remains to establish the existence of a sequence $t_{k} \rightarrow \infty$ such that $u\left(t_{k}\right)$ converges to some $u_{\infty} \in S(P)$. To this end we observe that the function $\Delta(t)$ is non-negative with $\int_{0}^{\infty} \Delta(\tau) d \tau<\infty$, so that we may find a sequence $t_{k} \rightarrow \infty$ such that $\Delta\left(t_{k}\right) \rightarrow 0$, and which may be further chosen so that $-\dot{u}\left(t_{k}\right) \in \partial f\left(u\left(t_{k}\right), r\left(t_{k}\right)\right)$. By extracting a subsequence if necessary we may also assume that $u\left(t_{k}\right)$ converges towards a certain $u_{\infty}$. Using Fenchel's inequality and the definition of $\Delta(t)$, we get

$$
f\left(u\left(t_{k}\right), r\left(t_{k}\right)\right) \leq f\left(x\left(r\left(t_{k}\right)\right), r\left(t_{k}\right)\right)+\Delta\left(t_{k}\right),
$$

so that, using the fact that $f\left(x\left(r\left(t_{k}\right)\right), r\left(t_{k}\right)\right) \rightarrow \inf \{f(x): x \in H\}$ and the conver- 
gence hypothesis $(C)$, we deduce that

$$
f\left(u_{\infty}\right) \leq \inf \{f(x): x \in H\}
$$

that is to say $u_{\infty} \in S(P)$, achieving the proof of our theorem.

In the infinite dimensional case we may also establish the weak convergence of $u(t)$ under an extra monotonicity assumption.

Theorem 4.2. In addition to the basic assumptions in $\S 2$, let us assume conditions $(C)$ and (6). Let us suppose further that for each $K \geq 0$ there exists $r_{K}>0$ such that $r \rightarrow f(x, r)$ is non-decreasing on $\left(0, r_{K}\right)$ for all $x \in B(0, K)$. Then, if $r(t)$ satisfies (8), the solution $u(t)$ of $(E V)$ converges weakly towards a particular solution $u_{\infty} \in S(P)$.

Proof. The proof is a slight modification of the previous one. As a matter of fact, the argument leading to the convergence of $|u(t)-\bar{u}|$ for all $\bar{u} \in S(P)$ was not based on finite dimensional arguments, so that we just need to modify the conclusion: we shall prove that every weak accumulation point of $u(t)$ belongs to the optimal solution set $S(P)$, so that the conclusion follows by invoking Opial's lemma [13].

On account of the assumption $(C)$, it suffices to show that $f(u(t), r(t))$ converges to $\inf \{f(x): x \in H\}$. As a matter of fact, since we have

$$
\int_{0}^{\infty}[f(u(\tau), r(\tau))-f(x(r(\tau)), r(\tau))] d \tau \leq \int_{0}^{\infty} \Delta(\tau) d \tau<\infty
$$

and since $f(x(r(t)), r(t)) \rightarrow \inf \{f(x): x \in H\}$, this reduces to showing that $f(u(t), r(t))$ converges, which is a direct consequence of [9, Proposition 1].

Remark 4.1. Clearly, by appropriately modifying the function $f$, one may also consider the case when there exists a function $\varphi: \mathbb{R}_{+} \rightarrow \mathbb{R}$ such that $\lim _{r \rightarrow 0^{+}} \varphi(r)$ exists and $r \rightarrow f(x, r)+\varphi(r)$ is non-decreasing for $r$ small.

Remark 4.2. Weak convergence of $u(t)$ in the infinite dimensional case was also established in [9, Theorem 2] under a weaker form of monotonicity (see assumption (A) in [9]). However, the analysis in that work is based on a fast parametrization condition which is of a different nature than (8): instead of controlling the speed of convergence through the bias of an optimal path $x(r)$, this speed is controlled in terms of the distance between the epigraphs of the approximating function $f(\cdot, r(t))$ and the limit function $f(\cdot)$, which is a strengthened form of Mosco-convergence (see assumption $\left(\mathrm{B}^{\prime}\right)$ in $\left.[9]\right)$.

\section{Application to the logarithmic BarRier in $(L P)$}

We present next the application of the previous results in the case of the logarithmic barrier function associated with the linear program

$$
\min \left\{c^{t} x: A x \leq b\right\}
$$

We suppose that the optimal solution set of $(L P)$ is nonempty and bounded, so that the $m \times n$ matrix $A$ has full rank $n(n<m)$.

The logarithmic barrier method is given by

$$
\min _{x \in P^{<}}\left\{c^{t} x-r \sum_{i=1}^{m} \ln \left(b_{i}-A_{i} x\right)\right\},
$$


where $r>0$ and $P^{<}:=\left\{x \in \mathbb{R}^{n}: A x<b\right\}$ is the domain of the barrier function $f(x, r):=c^{t} x-r \sum_{i=1}^{m} \ln \left(b_{i}-A_{i} x\right)$. The function $f(\cdot, r)$ is not defined outside $P^{<}$, and $f(x, r)$ tends to $+\infty$ when $x$ approaches the boundary of the feasible set of $(L P)$, which acts as a repulsive barrier that prevents one from violating the constraints.

It is known that $\left(L P_{r}\right)$ has a unique solution $x(r)$ which tends towards a particular point $x^{*} \in S(L P)$, called the analytic center, and that the function $x(r)$ is of class $C^{\infty}$ on $(0,+\infty)$ with $\left|\frac{d x}{d r}(r)\right| \leq L$ for a suitable constant $L$ and all $r$ close to 0 . This trajectory has been extensively studied in recent years because of its relevance to the so-called interior point methods (see $[3,11,12]$ and references therein).

The evolution equation $(E V)$ becomes in this case

$$
\left\{\begin{array}{l}
\dot{u}(t)=-\left[c+\sum_{i=1}^{m} \frac{r(t)}{b_{i}-A_{i} u(t)} A_{i}^{t}\right] \\
u(0)=u_{0}
\end{array}\right.
$$

where the initial value $u_{0}$ is supposed to belong to $P^{<}$. We begin by establishing the existence of a global solution of ( $L E V)$.

Proposition 5.1. There exists a unique solution $u(t)$ of the problem $(L E V)$, which is defined on the whole interval $[0,+\infty)$ and satisfies $u(t) \in P^{<}$for all $t \geq 0$. Moreover, $u(t)$ is of class $C^{\infty}$.

Proof. Let $u:[0, T) \rightarrow P^{<}$be a maximal solution of $(L E V)$ and suppose that $T<+\infty$. We shall prove that $\lim _{t \rightarrow T} u(t)$ exists and belongs to $P^{<}$, so that the existence and uniqueness of local solutions for $(L E V)$ contradict the maximality of $u$. From this we get simultaneously $T=+\infty$ and $u(t) \in P^{<}$for all $t \geq 0$.

Since the trajectory $x(r)$ satisfies the finite length assumption (6), we can prove that $u(t)$ stays bounded, just as in the first part of the proof of Theorem 4.1.

We observe next that $u(t)$ stays bounded away from the boundary of $P^{<}$, namely, there exists $\epsilon>0$ such that $u(t) \in P^{\epsilon}:=\left\{x: A_{i} x \leq b_{i}-\epsilon, i=1 \ldots m\right\}$ for all $t \in[0, T)$. Indeed, since $u(t)$ stays bounded we may find a constant $M$ such that $\sum_{i=1}^{m} \ln \left(b_{i}-A_{i} u(t)\right) \leq M$, and then a straightforward computation gives

$$
\frac{d}{d t} f(u(t), r(t))=-|\dot{u}(t)|^{2}+\frac{\partial f}{\partial r}(u(t), r(t)) \dot{r}(t) .
$$

Hence

$$
\frac{d}{d t} f(u(t), r(t)) \leq-\dot{r}(t) \sum_{i=1}^{m} \ln \left(b_{i}-A_{i} u(t)\right) \leq-M \dot{r}(t) .
$$

It follows that $f(u(t), r(t))+M r(t)$ is a decreasing function of $t$; thus $f(u(t), r(t))$ remains bounded from above for $t \in[0, T)$. From this we get easily that $u(t)$ must stay away from the boundary of the feasible polytope $P$, and by continuity we deduce the existence of $\epsilon$ with the required property.

To conclude the proof it suffices to remark that

$$
|\dot{u}(t)| \leq|c|+\sum_{i=1}^{m} \frac{r(t)}{b_{i}-A_{i} u(t)}\left|A_{i}\right| \leq|c|+\frac{r(0)}{\epsilon} \sum_{i=1}^{m}\left|A_{i}\right| \text { for all } t \in[0, T),
$$

from which we deduce the existence of $\lim _{t \rightarrow T} u(t)$ which belongs to $P^{\epsilon} \subset P^{<}$

We prove next that the solution $u(t)$ of $(L E V)$ converges when $t \rightarrow \infty$ towards an optimal solution $u_{\infty} \in S(L P)$. The arguments for proving this convergence differ depending on whether the function $r(t)$ is integrable on $[0, \infty)$ or not. 
Proposition 5.2. For every decreasing function $r(t)$ such that $r(t)$ goes to 0 when $t \rightarrow \infty$, and for every $u_{0} \in P^{<}$, the solution $u(t)$ of $(L E V)$ converges as $t \rightarrow \infty$ towards an optimal solution $u_{\infty} \in S(L P)$.

Proof. The equality

$$
h^{t} \nabla^{2} f(x, r) h=r \sum_{i=1}^{m} \frac{1}{\left(b_{i}-A_{i} x\right)^{2}}\left(A_{i} h\right)^{2}
$$

and the full rank of $A$ imply that condition $(L S C)$ holds with $\beta_{K}(r)=\alpha_{K} r$ for appropriate constants $\alpha_{K}$. Now, since $|d x / d r|$ is bounded we may apply Theorem 3.3 to conclude that when $\int_{0}^{\infty} r(\tau) d \tau=+\infty$ the trajectory $u(t)$ converges towards the analytic center $x^{*}$.

In the case $\int_{0}^{\infty} r(\tau) d \tau<+\infty$ the convergence of $u(t)$ will follow from Theorem 4.1 if we check (8). To this end let us consider a given $\bar{u} \in S(L P)$ and take $\epsilon>0$. Let us set $x_{\epsilon}(t):=x(r(t))+\epsilon\left(\bar{u}-x^{*}\right)$. We notice that $c^{t} x(r(t))=c^{t} x_{\epsilon}(t)$ and $A_{i} x(r(t))=A_{i} x_{\epsilon}(t)$ for $i \in I_{0}$, where $I_{0}:=\left\{i: A_{i} x=b_{i}\right.$ for all $\left.x \in S(L P)\right\}$. Then,

$$
f\left(x_{\epsilon}(t), r(t)\right)-f(x(r(t)), r(t))=r(t) \sum_{i \notin I_{0}}\left[\ln \left(b_{i}-A_{i} x(r(t))\right)-\ln \left(b_{i}-A_{i} x_{\epsilon}(t)\right)\right] .
$$

Since the trajectory $x(r)$ is bounded we may find a constant $K$ such that

$$
f\left(x_{\epsilon}(t), r(t)\right)-f(x(r(t)), r(t)) \leq r(t)\left[K-\sum_{i \notin I_{0}} \ln \left(b_{i}-A_{i} x_{\epsilon}(t)\right)\right] .
$$

Moreover, it is known that the analytic center $x^{*}$ belongs to the relative interior of $S(L P)$, which implies that $A_{i} x^{*}<b_{i}$ for all $i \notin I_{0}$. We may use this inequality to find $\alpha>0$ such that for $\epsilon$ close enough to 0 and all $t$ sufficiently large one has $b_{i}-A_{i} x_{\epsilon}(t) \geq \alpha$. Using this in combination with (10), we may find another constant $\tilde{K}$ such that

$$
f\left(x_{\epsilon}(t), r(t)\right)-f(x(r(t)), r(t)) \leq \tilde{K} r(t),
$$

from which it follows that $\lim _{t \rightarrow \infty} \Gamma_{\epsilon}(t)<+\infty$, as was to be proved.

This result shows that the conditions of fast and slow decay are complementary in the case of the log barrier for linear programming. We remark that this is not always the case, as observed in [7] for the exponential penalty. In the general case it remains as an open question to establish whether $\lim _{t \rightarrow \infty} u(t)$ exists for every parametrization $r(t)$ or not.

Concerning the results of $\S 3$ on the rate of convergence when $r(t)$ decays slowly, a straightforward application of Theorem 3.4 tell us that if the parametrization $r(t)$ is such that $\lim _{t \rightarrow \infty} \dot{r}(t) / r^{k+1}(t)=0\left(\right.$ e.g. $r(t)=1 / t^{\alpha}$ for $\left.\alpha<1 / k\right)$, then we have

$$
\lim _{t \rightarrow \infty} \frac{|u(t)-x(r(t))|}{r(t)^{k}}=0 .
$$

The particular case $k=1$ allows us to prove the convergence of some estimates of Lagrange multipliers for $(L P)$ associated with $u(t)$, namely

Proposition 5.3 (Convergence of Multipliers). Let $\lambda_{i}(t):=r(t) /\left(b_{i}-A_{i} u(t)\right)$ and suppose that $r(t)$ satisfies $\lim _{t \rightarrow \infty} \dot{r}(t) / r^{2}(t)=0$. Then $\lim _{t \rightarrow \infty} \lambda(t)$ exists, and it is an optimal solution for the dual problem $(D P)$ of $(L P)$. 
Proof. It suffices to notice that $r /\left(b_{i}-A_{i} x(r)\right)$ is known to converge towards a dual optimal solution $\lambda^{*} \in S(D P)$ (see [12]), and the condition $\lim _{t \rightarrow \infty} \dot{r}(t) / r^{2}(t)=0$ implies that $\lim _{t \rightarrow \infty}|u(t)-x(r(t))| / r(t)=0$.

\section{Continuous transition from fast to SlOW Decay}

We conclude by establishing a simple continuity result concerning the application that associates with a given parametrization $r(\cdot)$ the limit $u_{\infty}^{r}$ of the corresponding solution $u^{r}(t)$. More precisely, we establish that the limit $u_{\infty}^{r}$ converges towards $x^{*}$ when we pass from fast decay to slow decay.

As mentioned in the previous section, the convergence analysis under the assumptions of slow and fast decay may or may not cover all the possible decreasing functions $r(t)$. Therefore, we shall consider the set $\mathcal{L}$ of parametrizations $r(\cdot)$ decreasing to 0 such that the limit $u_{\infty}^{r}:=\lim _{t \rightarrow \infty} u^{r}(t)$ exists, where $u^{r}$ is the solution of $(E V)$ corresponding to $r$.

Theorem 6.1. In addition to the assumptions in $\S 2$ let us suppose that $(S C)$ holds with $\beta(\cdot)$ measurable and bounded over bounded sets, and that the optimal trajectory $x(r)$ satisfies the finite length assumption (6). Let $\left\{r_{k}: k \in \mathbb{N}\right\}$ be a sequence in $\mathcal{L}$ which converges pointwise towards some $r \in \mathcal{L}$. Let $u^{k}(t)$ be the solution of $(E V)$ corresponding to $r_{k}(t)$, and denote

$$
u_{\infty}^{k}=\lim _{t \rightarrow \infty} u^{k}(t) .
$$

If the sequence $r_{k}$ is such that $\int_{0}^{\infty} \beta\left(r_{k}(\tau)\right) d \tau \rightarrow \infty$ when $k \rightarrow \infty$, then

$$
\lim _{k \rightarrow \infty} u_{\infty}^{k}=x^{*} .
$$

Proof. Using the estimate

$$
\left|u^{k}(t)-x\left(r_{k}(t)\right)\right| \leq e^{-E_{k}(t)}\left|u_{0}-x\left(r_{k}(0)\right)\right|-\int_{0}^{t} e^{E_{k}(\tau)-E_{k}(t)}\left|\frac{d x}{d r}\left(r_{k}(\tau)\right)\right| \dot{r}_{k}(\tau) d \tau,
$$

where $E_{k}(t):=\int_{0}^{t} \beta\left(r_{k}(\xi)\right) d \xi$ (see [2, Theorem 3.2]), and letting $t \rightarrow \infty$, we obtain

$$
\left|u_{\infty}^{k}-x^{*}\right| \leq e^{-E_{k}(\infty)}\left|u_{0}-x\left(r_{k}(0)\right)\right|-\int_{0}^{\infty} e^{E_{k}(\tau)-E_{k}(\infty)}\left|\frac{d x}{d r}\left(r_{k}(\tau)\right)\right| \dot{r}_{k}(\tau) d \tau .
$$

Since by hypothesis we have $E_{k}(\infty) \rightarrow \infty$, the first term on the right-hand side goes to zero when $k \rightarrow \infty$. In order to show that the integral term also goes to 0 we split it in two pieces: for each $M>0$ we have

$$
\begin{aligned}
& -\int_{0}^{\infty} e^{E_{k}(\tau)-E_{k}(\infty)}\left|\frac{d x}{d r}\left(r_{k}(\tau)\right)\right| \dot{r}_{k}(\tau) d \tau \\
& \quad \leq-e^{E_{k}(M)-E_{k}(\infty)} \int_{0}^{M}\left|\frac{d x}{d r}\left(r_{k}(\tau)\right)\right| \dot{r}_{k}(\tau) d \tau-\int_{M}^{\infty}\left|\frac{d x}{d r}\left(r_{k}(\tau)\right)\right| \dot{r}_{k}(\tau) d \tau .
\end{aligned}
$$

Letting $k \rightarrow \infty$ for $M$ fixed, we see that the quantity

$$
E_{k}(M)-E_{k}(\infty)=-\int_{M}^{\infty} \beta\left(r_{k}(\xi)\right) d \xi
$$

diverges to $-\infty$, so that the first term on the right converges to 0 while the second term goes to $\int_{0}^{r(M)}\left|\frac{d x}{d r}\right| d r$. Since for $M \rightarrow \infty$ the latter can be made as small as desired, we deduce that the integral on the left converges to 0 when $k \rightarrow \infty$, and the proof is complete. 
Remark 6.1. The previous result remains valid if the initial point $u_{0}$ is also perturbed for each $k$, provided the sequence of initial conditions $u_{0}^{k}$ remains bounded.

\section{REFERENCES}

[1] Attouch H. and Damlamian A., "Strong solutions for parabolic variational inequalities", Journal of Nonlinear Analysis, Vol. 2, pp.329-353, 1978. MR 80a:47093

[2] Atтouch H. And Cominetti R., "A dynamical approach to convex minimization coupling approximation with the steepest descent method", Journal of Differential Equations, Vol. 128, pp. 519-540, 1996. MR 97e:90112

[3] BAYER D. AND Lagarias J.C., "The nonlinear geometry of linear programming (parts I and II)", Transactions of the AMS, Vol. 314, pp. 499-526, 527-581, 1989. MR 90h:90098

[4] BrÉzis H., Asymptotic behavior of some evolution systems: Nonlinear Evolution Equations, Academic Press, 1978, pp. 141-154. MR 80f:47060

[5] BRUCK R.E., "Asymptotic convergence of nonlinear contraction semigroups in Hilbert space", Journal of Functional Analysis, Vol. 18, pp.15-26, 1975. MR 51:13780

[6] Cominetti R., "Asymptotic convergence of Euler's method for the exponential penalty in linear programming", Journal of Convex Analysis, Vol. 2, pp. 145-152, 1995. MR 96h:90061

[7] Cominetti R. and San Martín J., "Asymptotic analysis for the exponential penalty trajectory in linear programming", Mathematical Programming, Vol. 67, pp. 169-187, 1994. MR 95h:90062

[8] Ekeland I. And Temam R., Convex analysis and variational problems, North-Holland, Amsterdam, 1976. MR 57:3931

[9] Furuya H., Miyashiba K. and Kenmochi N., "Asymptotic behavior of solutions to a class of nonlinear evolution equations", Journal of Differential Equations, Vol. 62, pp.73-94, 1986. MR 87k:34105

[10] Kenmochi N., "Solvability of nonlinear evolution equations with time-dependent constraints and applications", Bull. Fac. Ed. Chiba Univ., Vol. 30, pp. 1-87, 1981.

[11] MCLinden L., "An analogue of Moreau's proximation theorem with applications to the nonlinear complementarity problem", Pacific J. Math., Vol. 88, pp. 101-161, 1980. MR 82k:90118

[12] Megiddo N., "Pathways to the optimal set in linear programming", in Progress in Mathematical Programming: Interior-Point and Related Methods, Springer-Verlag, Berlin, pp. 131-158, 1989. MR 90c:90147

[13] Opial Z., "Weak convergence of the sequence of successive approximations for nonexpansive mappings", Bulletin of the American Mathematical Society, Vol. 73, pp. 591-597, 1967. MR 35:2183

[14] Rockafellar R.T., Convex Analysis, Princeton University Press, 1970. MR 43:445

[15] Tikhonov A. And Arsenine V., Méthodes de résolution de problèmes mal posés, Mir, Moscow, 1974. MR 56:13605b

[16] Torralba D., Convergence épigraphique et changements d'échelle en analyse variationnelle et optimisation, Thesis, Université de Montpellier II, 1996.

Universidad de Chile, Casilla 170/3, Correo 3, Santiago, Chile.

E-mail address: rcominet@dim.uchile.cl 